\title{
The impact of suspension control on the controllability of the lateral vehicle dynamics
}

\author{
Péter Gáspár, Balázs Németh, József Bokor, Olivier Sename, Luc Dugard
}

\begin{abstract}
Since there is a coupling between lateral and vertical dynamics, the interactions between control components must be taken into consideration. The paper presents the effects of vertical load variations on the controlled invariant set of the steering system. In the model the nonlinear characteristics of the tire force are approximated by the polynomial form. The analysis is based on Sum-of-Squares programming method and parameter-dependent polynomial control Lyapunov functions. The Maximum Controlled Invariant Sets of the steering as a function of vertical loads are illustrated through a simulation example. The results of the analysis are built into the control design of the suspension system. A semi-active suspension system using preview control is applied. The operation of the controller is illustrated through simulation examples.
\end{abstract}

\section{INTRODUCTION AND MOTIVATION}

The coupling between the lateral and vertical dynamics is influenced by the changes of the vertical load variation and the effects of the suspension actuator and the steering system. The lateral force, which is a function of the vertical load, depends on the presence of the slip angle and the cornering stiffness, see [1], [2], [3]. The vertical load has a static component due to gravity and a dynamic component due to road unevennesses and the vertical motions of both the sprung mass and the unsprung masses. Thus, there is a relationship between the lateral force and the dynamic component of the vertical load.

The paper presents the effects of vertical load variations on the controlled invariant sets of the steering system. Using the Sum-of-Squares (SOS) programming method and parameterdependent polynomial control Lyapunov functions the Maximum Controlled Invariant Sets of the steering as a function of vertical loads are calculated. The SOS method has been elaborated in the last decade for control purposes, see e.g., [4], [5]. The paper also presents the performances of the suspension system, in which the results of the SOS analysis are exploited. Since the purpose is to reduce the variations of the lateral force during maneuvers, it is necessary that the

P. Gáspár and J. Bokor are with Institute for Computer Science and Control, Hungarian Academy of Sciences and MTA-BME Control Engineering Research Group, Budapest, Hungary. E-mail: [gaspar.peter;bokor.jozsef]@sztaki.mta.hu

B. Németh is with Systems and Control Laboratory, Institute for Computer Science and Control, Hungarian Academy of Sciences, Kende u. 1317, H-1111 Budapest, Hungary. E-mail: [bnemeth]@sztaki.mta.hu

O. Sename and L. Dugard are with GIPSA-lab, Grenoble Institute of Technology, 11 rue des mathématiques, 38402 Saint Martin d'Heres, France. E-mail: [olivier.sename;luc.dugard]@gipsa-lab.grenoble-inp.fr

The research was supported by the National Research, Development and Innovation Fund through the project "SEPPAC: Safety and Economic Platform for Partially Automated Commercial vehicles" (VKSZ 14-1-20150125). This paper was partially supported by the János Bolyai Research Scholarship of the Hungarian Academy of Sciences. dynamic component of the vertical load should be kept as small as possible.

The contribution of the paper is to justify the necessity of the integration of the lateral and the vertical dynamics, thus the integration of the steering and suspension controls. Moreover, the results of the lateral analysis utilizing the nonlinear characteristics of the tire are built into the design of semi-active suspension control through the performance specifications and weighting strategy.

The structure of the paper is the following. Section II presents the relationship between lateral and vertical dynamics. Although the bicycle model describes the vehicle dynamics in the plane, it represents vertical dynamics through cornering stiffness as well. Section III analyzes the effects of vertical load variations on the maximum controlled invariant set. Section IV presents the performance specifications of the suspension system. In Section $\mathrm{V}$ the operation of the semiactive suspension system is illustrated.

\section{THE BACKGROUND OF SUSPENSION AND STEERING INTEGRATION}

In the interaction between lateral and vertical dynamics, consequently the interaction between the steering system and the suspension system, the vertical tire load plays a significant role, see e.g, [6]. From the suspension point of view the vertical tire load can be modified through the suspension control. From the steering point of view, the values of the lateral tire force $\mathcal{F}$ are fundamentally determined by the vertical tire load $F_{t}$.

\section{Polynomial form of the lateral tire force}

The lateral tire force $\mathcal{F}$ depends on the side-slip angle $\alpha$ and the vertical tire load $F_{t}$, thus $\mathcal{F}=\mathcal{F}\left(\alpha, F_{t}\right)$. The relation is defined by a polynomial description as a function of the vertical tire force, in which the nonlinearities of the tire characteristics are considered in a given operation range [7] in the following form:

$$
\mathcal{F}\left(\alpha, F_{t}\right)=c_{1}\left(F_{t}\right) \alpha+. .+c_{n}\left(F_{t}\right) \alpha^{n}=\sum_{k=1}^{n} c_{k}\left(F_{t}\right) \alpha^{k},
$$

where the coefficient function $c_{k}\left(F_{t}\right)$ has a polynomial form:

$$
c_{k}\left(F_{t}\right)=d_{1} F_{t}+d_{2} F_{t}^{2}+. .+d_{m} F_{t}^{m}=\sum_{j=1}^{m} d_{j} F_{t}^{j}
$$

The relationship between the vertical tire force $F_{t}$, the side-slip angle $\alpha$ and the lateral tire force $\mathcal{F}\left(\alpha, F_{t}\right)$ is illustrated in Figure 1. A suitable approximation of tire force 


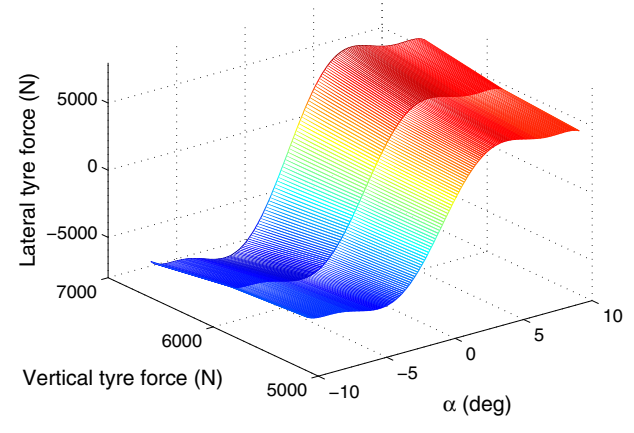

Fig. 1. Vertical load dependence

characteristics between the slip region $\alpha=-12^{\circ} \ldots+12^{\circ}$ can be achieved by selecting $n=10$ and $m=2$. Note that the nonlinear tire characteristics can also be modeled by other methods, see e.g., [2], [8].

In the following, the variation of the vertical tire load is illustrated through a simulation example in Figure 2. The car is traveling on the road and during a maneuver a bump also disturbs the motion. These excitations result in significant effects on the vertical dynamics. Consequently, the side-slip angle and the yaw rate are also significantly modified due to the function $\mathcal{F}\left(\alpha, F_{t}\right)$.

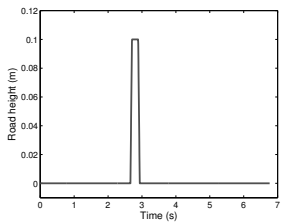

(a) Road profile

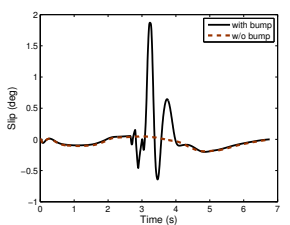

(c) Side slip

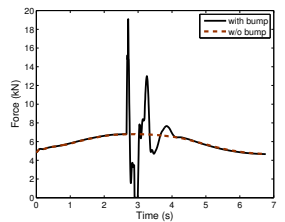

(b) Vertical tire load

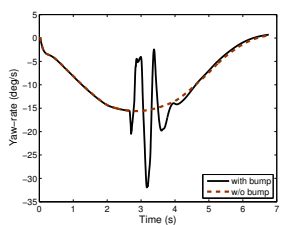

(d) Yaw rate
Fig. 2. Simulation results - bump on the road

\section{Nonlinear tire characteristics in lateral vehicle dynamics}

Lateral vehicle dynamics is based on a two-wheeled model, which is shown in Figure 3. In the following a polynomial form is applied to the nonlinear tire characteristics.

$$
\begin{aligned}
& J \ddot{\psi}=\mathcal{F}_{1}\left(\alpha_{1}, F_{t, 1}\right) l_{1}-\mathcal{F}_{2}\left(\alpha_{2}, F_{t, 2}\right) l_{2} \\
& m v(\dot{\psi}+\dot{\beta})=\mathcal{F}_{1}\left(\alpha_{1}, F_{t, 1}\right)+\mathcal{F}_{2}\left(\alpha_{2}, F_{t, 2}\right)
\end{aligned}
$$

where $m$ is the mass of the vehicle, $J$ is yaw-inertia, $l_{1}$ and $l_{2}$ are geometric parameters. $\beta$ is the side-slip angle of the chassis, $\dot{\psi}$ is the yaw rate. $\mathcal{F}_{1}\left(\alpha_{1}, F_{t, 1}\right)$ and $\mathcal{F}_{2}\left(\alpha_{2}, F_{t, 2}\right)$ represent the lateral tire forces at the front and the rear according to (1). The side-slip angles of the front and rear axles are approximated:

$$
\alpha_{1}=\delta-\beta-\frac{\dot{\psi} l_{1}}{v}, \quad \alpha_{2}=-\beta+\frac{\dot{\psi} l_{2}}{v}
$$

where $\delta$ is the front wheel steering. In the following the state space representation, in which the state variables are $\alpha_{1}$ and $\alpha_{2}$ is formed.

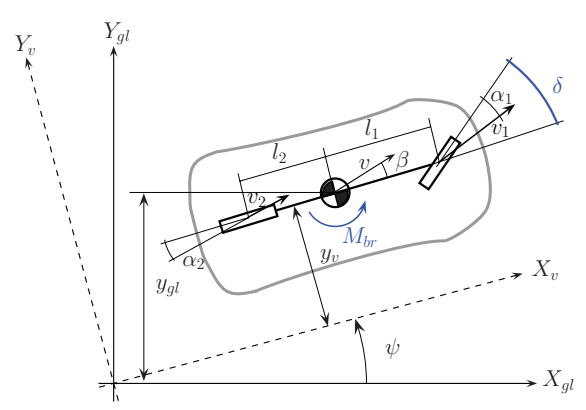

Fig. 3. Lateral vehicle model

Based on (3) and (4) the vehicle model is reformulated:

$$
\begin{aligned}
\dot{\alpha}_{2}-\dot{\alpha}_{1} & =\frac{l_{1}+l_{2}}{J v}\left[\mathcal{F}_{1}\left(\alpha_{1}, F_{t, 1}\right)\left(\alpha_{1}\right) l_{1}-\mathcal{F}_{2}\left(\alpha_{2}, F_{t, 2}\right) l_{2}\right] \\
& -\nu \delta \\
\dot{\alpha}_{1} l_{2}+\dot{\alpha}_{2} l_{1} & =v\left(\alpha_{2}-\alpha_{1}\right)+\left(v+l_{2} \nu\right) \delta- \\
& -\frac{l_{1}+l_{2}}{m v}\left[\mathcal{F}_{1}\left(\alpha_{1}, F_{t, 1}\right)+\mathcal{F}_{2}\left(\alpha_{2}, F_{t, 2}\right)\right]
\end{aligned}
$$

The parameter $\nu$ is introduced, which represents the relationship between the maximum steering value and the variation speed of $\delta$. The signal $\dot{\delta}$ is modeled as $\dot{\delta} \cong \nu \cdot \delta$, see [7]. Since $\max \delta$ is a given fixed limit at the actuator analysis, a high $\nu$ value represents a fast-changing steering signal, while a slow-changing steering signal is modeled with a low $\nu$. Then the polynomial state-space representation of the system is formulated as follows:

$$
\dot{x}=\left[\begin{array}{c}
\dot{\alpha}_{1} \\
\dot{\alpha}_{2}
\end{array}\right]=\left[\begin{array}{c}
\mathrm{f}_{1}\left(\alpha_{1}, \alpha_{2}, F_{t, 1}, F_{t, 2}\right) \\
\mathrm{f}_{2}\left(\alpha_{1}, \alpha_{2}, F_{t, 1}, F_{t, 2}\right)
\end{array}\right]+\left[\begin{array}{c}
h_{1} \\
h_{2}
\end{array}\right] \delta
$$

\section{THE EFFECTS OF VERTICAL LOAD VARIATION ON THE MAXIMUM CONTROLLED INVARIANT SET}

In the paper the SOS programming method is applied for the analysis of the effects of the vertical load variation on lateral dynamics. The SOS method has been elaborated in the last decade for control purposes. Important theorems in SOS programming, such as the application of Positivstellensatz, were proposed in [4]. Thus, the convex optimization methods can be used to find appropriate polynomials of the SOS problem, see [9]. Sufficient conditions for the solutions to nonlinear control problems, which were formulated in terms of state-dependent Linear Matrix Inequalities (LMI), were formed by [5]. The goal of the analysis is to show the effect of vertical load variation on the size of the sets. 


\section{Theoretical background}

The following definitions and theorems are essential to understand SOS programming [10]. The state-space representation of the system is given in the following form, see (6):

$$
\dot{x}=f\left(F_{t, 1}, F_{t, 2}, x\right)+g u
$$

where the state vector of the system is $x^{T}=\left[\alpha_{1}, \alpha_{2}\right]$. The expression $f\left(F_{t, 1}, F_{t, 2}, x\right)$ is a matrix, which incorporates smooth polynomial functions and $f\left(F_{t, 1}, F_{t, 2}, 0\right)=0$. In the next analysis the control input is $u=\delta$. In the following analysis the vertical loads $F_{t, 1}, F_{t, 2}$ are fixed, thus $f\left(F_{t, 1}, F_{t, 2}, x\right)=f(x)$ depends on the state vector $x$.

The global asymptotical stability of the system at the origin is guaranteed by the existence of the Control Lyapunov Function of the system defined as follows [11]:

Definition 1: A smooth, proper and positive-definite function $V: \mathbb{R}^{n} \rightarrow \mathbb{R}$ is a Control Lyapunov Function for the system if

$$
\inf _{u \in \mathbb{R}}\left\{\frac{\partial V}{\partial x} f(x)+\frac{\partial V}{\partial x} g \cdot u\right\}<0
$$

for each $x \neq 0$.

Thus, it is necessary to find a Control Lyapunov Function $V$ which meets the following set emptiness conditions:

$$
\begin{aligned}
& \left\{-\frac{\partial V}{\partial x} g-\epsilon \geq 0,1-V(x) \geq 1, l_{1}(x) \neq 0\right. \\
& \left.\frac{\partial V}{\partial x} f(x)+\frac{\partial V}{\partial x} g \cdot \bar{u} \geq 0, \frac{\partial V}{\partial x} f(x)+\frac{\partial V}{\partial x} g \cdot \bar{u} \neq 0\right\}=\emptyset \\
& \left\{\frac{\partial V}{\partial x} g-\epsilon \geq 0,1-V(x) \geq 1, l_{2}(x) \neq 0,\right. \\
& \left.\frac{\partial V}{\partial x} f(x)-\frac{\partial V}{\partial x} g \cdot \bar{u} \geq 0, \quad \frac{\partial V}{\partial x} f(x)-\frac{\partial V}{\partial x} g \cdot \bar{u} \neq 0\right\}=\emptyset
\end{aligned}
$$

Note that the relations in the third inequality are inverted to guarantee the emptiness of the sets. The role of $l_{1,2}(x) \neq 0$ is to guarantee the condition $x \neq 0$ in (1). $l_{1,2}(x)$ is chosen as a positive definite polynomial [10].

Since it is necessary to find the Maximum Controlled Invariant Sets, another set emptiness condition is also defined to improve the efficiency of the method [10]:

$$
\{p(x) \leq \beta, V(x) \geq 1, V(x) \neq 1\}=\emptyset
$$

where $p \in \Sigma_{n}$ is a fixed and positive definite function. $\beta$ defines a $P_{\beta}:=\left\{x \in \mathbb{R}^{n} \mid p(x) \leq \beta\right\}$ level set, which is incorporated in the actual Controlled Invariant Set. Thus, the maximization of $\beta$ enlarges $P_{\beta}$ together with the Controlled Invariant Set.

In the next step the set-emptiness conditions are reformulated to SOS conditions based on the generalized Sprocedure. In the formulation $\Sigma_{n}$ represents SOS.

Theorem 1: Generalized S-Procedure: Given symmetric matrices $\left\{p_{i}\right\}_{i=0}^{m} \in \mathcal{R}_{n}$. If there exist nonnegative scalars $\left\{s_{i}\right\}_{i=1}^{m} \in \Sigma_{n}$ such that

$$
p_{0}-\sum_{i=1}^{m} s_{i} p_{i} \succeq q
$$

with $q \in \Sigma_{n}$, then

$$
\bigcap_{i=1}^{m}\left\{x \in \mathbb{R}^{n} \mid p_{i}(x) \geq 0\right\} \subseteq\left\{x \in \mathbb{R}^{n} \mid p_{0}(x) \geq 0\right\}
$$

The related set emptiness question is whether

$$
\begin{array}{r}
W:=\left\{x \in \mathbb{R}^{n} \mid p_{1}(x) \geq 0, \ldots, p_{m}(x) \geq 0,\right. \\
\left.-p_{0}(x) \geq 0, p_{0}(x) \neq 0\right\}
\end{array}
$$

is empty.

The conditions (9) and (10) have the same structure as (13), therefore the reconstruction can be carried out (11). Thus, the next optimization problem is formed to find the maximum Controlled Invariant Set:

$$
\max \beta
$$

over $s_{i} \in \Sigma_{n}, i=[1 \ldots 5] ; V, p_{1}, p_{2} \in \mathcal{R}_{n} ; V(0)=0$ such that

$$
\begin{aligned}
& -\left(\frac{\partial V}{\partial x} f(x)+\frac{\partial V}{\partial x} g \cdot \bar{u}\right)-s_{1}\left(-\frac{\partial V}{\partial x} g-\epsilon\right)- \\
& -s_{2}(1-V)-p_{1} l_{1} \in \Sigma_{n} \\
& -\left(\frac{\partial V}{\partial x} f(x)-\frac{\partial V}{\partial x} g \cdot \bar{u}\right)-s_{3}\left(\frac{\partial V}{\partial x} g-\epsilon\right)- \\
& -s_{4}(1-V)-p_{2} l_{2} \in \Sigma_{n} \\
& -\left(s_{5}(\beta-p)+(V-1)\right) \in \Sigma_{n}
\end{aligned}
$$

\section{Maximum Controlled Invariant Sets of the steering}

The result of the optimization (15) is the Maximum Controlled Invariant Set $V(x)=1$, which is related to fixed vertical loads $F_{t, 1}, F_{t, 2}$. The set depicts the states, from which the system can be stabilized using the control input $\underline{u} \leq u \leq \bar{u}$. The size of the computed set is determined by $F_{t, 1}, F_{t, 2}$ through the lateral forces, see (1). In the following an analysis is shown which illustrates the effect of the vertical load on the size of the set, see Figure 4.

In the examination the speed of the vehicle is fixed at $v=$ $20 \mathrm{~m} / \mathrm{s}$ and the range of the steering control input is between $-12^{\circ} \leq \delta \leq 12^{\circ}$. The Maximum Controlled Invariant Sets are computed at fixed but different vertical loads on the front and the rear wheels as functions of the side-slip angles at the front and rear. The fixed vertical loads are $F_{t, i}=\{3000 \mathrm{~N}$, $4000 N, 5000 N\}$. The analysis shows that the vertical loads significantly affect the size of the invariant sets, which is shown by ellipsoids in the plot. If the value $F_{t, i}$ decreases, the size of the invariant sets in which the vehicle can be stabilized also decreases.

Another contribution of the analysis comes from the relationship between the vertical loads at the front and rear $F_{t, 1}$ and $F_{t, 2}$. If $F_{t, 2}$ is fixed, for example $F_{t, 2}=4000 N$, the sizes of the invariant sets vary slightly with different $F_{t, 1}$. However, if $F_{t, 1}$ is fixed, for example $F_{t, 1}=4000 N$, the 


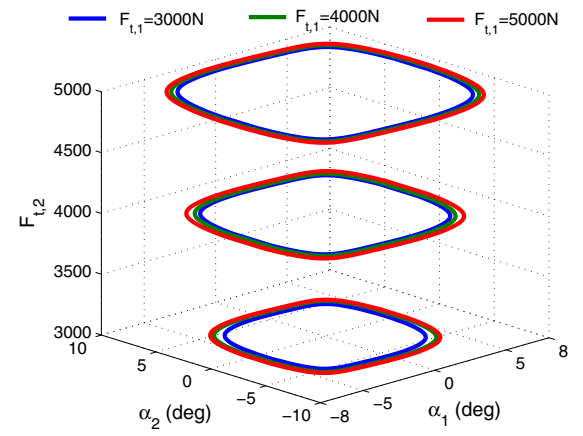

Fig. 4. Maximum Controlled Invariant Sets of the steering

sizes of the invariant sets vary significantly with different $F_{t, 2}$. It follows that the variation of the vertical load on the rear axle has a more significant impact on the lateral stability of the vehicle than that of the front axle.

Note that the SOS-based analysis can be performed also in the entire load range $F_{t, \min } \leq F_{t} \leq F_{t, \max }$. The SOS method results in robust Maximum Controlled Invariant Sets, in which the variation of the vertical load can be considered as uncertainty, see [12].

\section{Performance analysis and control design of THE SUSPENSION}

Based on the Maximum Controlled Invariant Sets of the steering the purpose of the suspension control design is to reduce the vertical tire load variations $F_{t}$ and/or avoid significant changes in $F_{t}$. Thus, the vertical tire load variation is incorporated in the performance criterion of the suspension control.

\section{Analysis of suspension performances}

In the paper the control design of the suspension system is based on the quarter-car model, which is modeled by the following two-force equations:

$$
\begin{aligned}
m_{s} \ddot{z}_{s} & =-k_{s}\left(z_{s}-z_{u s}\right)-b_{s}\left(\dot{z}_{s}-\dot{z}_{u s}\right)+F_{s} \\
m_{u s} \ddot{z}_{u s} & =k_{s}\left(z_{s}-z_{u s}\right)+b_{s}\left(\dot{z}_{s}-\dot{z}_{u s}\right)-F_{s}-k_{t}\left(z_{u s}-w\right)
\end{aligned}
$$

where $m_{s}, m_{u s}$ represent the sprung and unsprung masses, $k_{s}, b_{s}$ are the suspension stiffness and damping parameters, $k_{t}$ is the tire stiffness. $w$ is the external excitation caused by the road, $z_{s}, z_{u s}$ are the vertical displacements of the sprung and unsprung masses, while the control input of the system is the suspension force actuation $F_{s}$.

The vertical load minimization is one of the performance signals, which is expressed by the following form $F_{t}=$ $k_{t}\left(z_{u s}-w\right)$. This minimization shows that the displacement of $z_{u s}$ follows the road profile $w$ and it also guarantees that the vehicle remains on the track in all maneuvers. It is a tracking performance problem:

$$
z_{1}=z_{u s}-w \quad\left|z_{1}\right| \rightarrow \min
$$

The difficulty of this problem is that $w$ is an unknown disturbance. The design of the suspension systems is based on preview control, in which the road disturbance is assumed to be measured or estimated.

In the conventional design of the suspension system passenger comfort, which is expressed by the vertical acceleration of the sprung mass, is another performance signal:

$$
z_{2}=\ddot{z}_{s} \quad\left|z_{2}\right| \rightarrow \min
$$

is a good choice, as shown below. The vertical acceleration of the sprung mass is formulated using (16a), in which $F_{s}$ is expressed by equation (16b):

$$
\begin{aligned}
z_{2} & =-\frac{k_{s}}{m_{s}}\left(z_{s}-z_{u s}\right)-\frac{b_{s}}{m_{s}}\left(\dot{z}_{s}-\dot{z}_{u s}\right)+\frac{F_{s}}{m_{s}} \\
& =-\left[\frac{k_{t}}{m_{s}} z_{1}+\frac{m_{u s}}{m_{s}} \ddot{z}_{u s}\right]
\end{aligned}
$$

The relationship between the performances shows that $z_{2}$ incorporates the required performance $z_{1}$ and the acceleration of the unsprung mass. It also shows that the minimization of $\left|z_{2}\right|$ does not guarantee the vertical load minimization without applying additional energy to the system. This is the background of the trade-off between road holding and passenger comfort.

In the control design a semi-active suspension system is applied. The summary of the semi-active suspension control considering the comfort criterion is presented in [13], [14]. Sky-Hook and clipped control design laws based on model predictive control technique are used in [15]. LPV-based robust control design methods to improve the motion of the chassis are found in [16].

The control force $F_{s}$ of a magneto-rheological semi-active suspension system is formed as follows:

$$
\begin{aligned}
F_{s} & =k_{0}\left(z_{s}-z_{u s}\right)+c_{0}\left(\dot{z}_{s}-\dot{z}_{u s}\right)+ \\
& +f_{I} \tanh \left(k_{1}\left(z_{s}-z_{u s}\right)+c_{1}\left(\dot{z}_{s}-\dot{z}_{u s}\right)\right)
\end{aligned}
$$

where $c_{0}, c_{1}, k_{0}$ and $k_{1}$ are constant parameters and $0 \leq$ $f_{I, \min } \leq f_{I} \leq f_{I, \max }$ is the controllable force coefficient, which varies according to the electrical current $I$ in the coil, see [16]. The control task must be performed with a control signal as small as possible. Thus, the control input $z_{3}=f_{I}$ is also a performance signal.

Using (16) and (20) the vehicle model is formed as

$$
\dot{x}_{s}=A_{s} x_{s}+B_{s, 1} w+B_{s, 2}\left(\rho_{1}\right) u_{s}
$$

where $\rho_{1}=\tanh \left(k_{1}\left(z_{s}-z_{u s}\right)+c_{1}\left(\dot{z}_{s}-\dot{z}_{u s}\right)\right)$ is a scheduling variable of the system. The control input is $u_{s}=$ $f_{I}$, the performance output vector is $z_{s}=\left[\begin{array}{ll}z_{1} & z_{3}\end{array}\right]^{T}$ and the measured output vector is $y_{s}=\left[\begin{array}{lll}z_{s}-z_{u s} & \dot{z}_{s}-\dot{z}_{u s} & w\end{array}\right]^{T}$. Note that the model can be transformed into another form, in which $A_{s}$ depends on $\rho_{1}$, see [16].

\section{LPV-based control design of the suspension}

In the control design the minimization of vertical load variation $z_{1}$ is in the focus. Several weighting functions are built in the closed-loop interconnection structure, see Figure 5. The role of these weights is to scale the input and output signals and find a trade-off between the performances. The 
weight $W_{z_{1}}$ applies to the performance $z_{1}$, the weight $W_{z_{3}}$ applies to the control input, while the weight $W_{w}$ scales the road excitation signal.

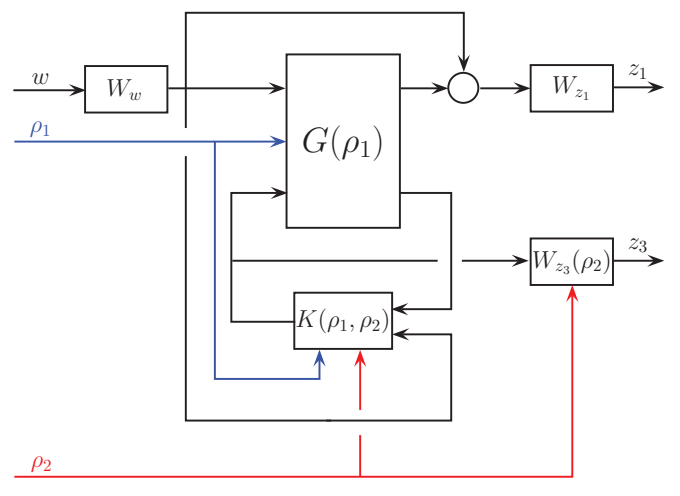

Fig. 5. Closed-loop interconnection structure

The results of the SOS-based controlled invariant sets are incorporated in the control design. They are the operation range of the controllers and the parameters of the weighting functions applied in the closed-loop interconnection structure. In the control design the requirement of the vertical load variation is fulfilled by the appropriate selection of $W_{z_{1}}$. The weight is defined as follows:

$$
W_{z_{1}}=\frac{A_{1} s+A_{0}}{T_{1} s+T_{0}}
$$

where $A_{1}, A_{0}, T_{1}$ and $T_{0}$ are design parameters, which guarantee the main performance. The ratio of $T_{0} / A_{0}$ represents a bound of the steady-state error of $z_{1}$. Thus, the ratio $A_{0} / T_{0}$ is chosen to be a high value. Moreover, if the maximum vertical load variation is defined as $\Delta F_{t}$, then the maximum variation of the tire compression is $\Delta z_{1}=\Delta F_{t} / k_{t}$. In practice, $W_{z_{1}}$ must guarantee that $\left|z_{1}\right| \leq \Delta z_{1}$. Thus, it is formulated in the high frequency range as the ratio $A_{1} / T_{1}>\Delta z_{1}$.

In the case of the semi-active suspension the control input $u_{s}=f_{I}$ has physical limits, which results from the actuator construction. The input saturation of the system in the design through the parameter-dependent weighting function $W_{z_{3}}\left(\rho_{2}\right)$ is considered, where $\rho_{2}$ is a scheduling variable. The defined scheduling variable $\rho_{2}$ is selected based on the operation range of the actuator. A possible selection rule is illustrated in Figure 6. $u_{s, i} \mathrm{~s}$ are design parameters related to $f_{I, \min }, f_{I, \max }$. A parameter-dependent weight $W_{z_{3}}\left(\rho_{2}\right)=W_{0, z_{3}} / \rho_{2}$ is applied in the control design. When $u_{s}$ is outside its operation range, then $\rho_{2}=\rho_{2, \min }$ is selected to penalize the input saturation.

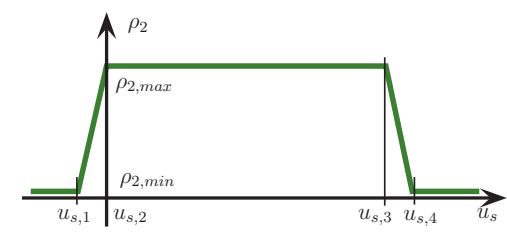

Fig. 6. Computation of scheduling variable $\rho_{2}$
The control design is based on the LPV method that uses parameter-dependent Lyapunov functions, see [17], [18].

\section{Simulation example}

The operations of the semi-active suspensions are shown through the simulation examples. During the simulation a cornering maneuver with constant steering angle is performed. In the interval $0.8 \ldots 1.9 \mathrm{~s}$ a bump, while in the rest of the road section random noise disturbances are found. Two different control strategies are used in the simulations: the suspension $K_{S, z_{1}}$ guarantee the minimization of performance $z_{1}$, while the suspension $K_{S, z_{2}}$ guarantee the minimization of performance $z_{2}$.

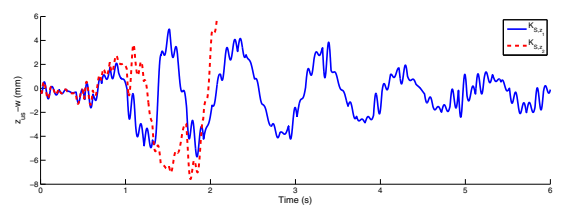

(a) Performance $z_{1}$

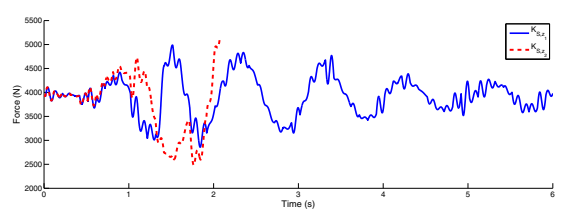

(b) Vertical load $F_{t}$

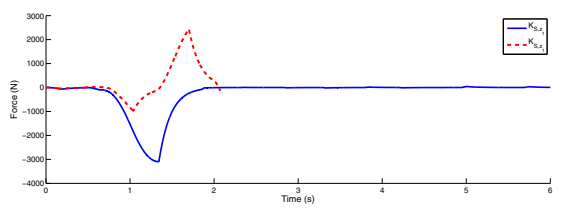

(c) Control input $F_{s}$

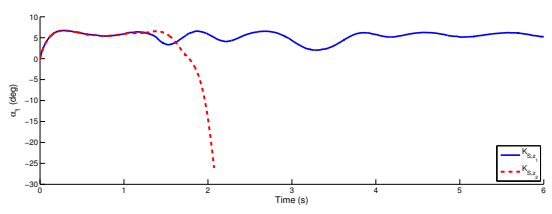

(d) Side-slip angle $\alpha_{1}$

Fig. 7. Simulation results

Figure 7 presents the time responses of the semi-active suspension controls. The road excitation results in a significant variation in the tire compression and the vertical load, see Figures 7(a)-7(b). However, the same contribution to $F_{t}$ is yielded: $K_{S, z_{1}}$ is able to minimize $F_{t}$, while $K_{S, z_{2}}$ results in higher amplitude. It is the consequence of the different $F_{s}$, as shown in Figure 7(c). The difference in the amplitude has an impact on the lateral dynamics, as shown in Figures 7(d). The results indicate that the reduced $F_{t}$ between $1 \ldots 2 s$ leads to significant change in lateral dynamics. The slip angles increase to critical values, e.g. $\alpha_{2}=-70^{\circ}$, which is hazardous.

Figure 8 shows the lateral side-slip angles $\alpha_{2}$ as function $\alpha_{1}$. The time responses of the controlled system which apply the controller $K_{S, z_{1}}$ remain a bounded plane, which shows 


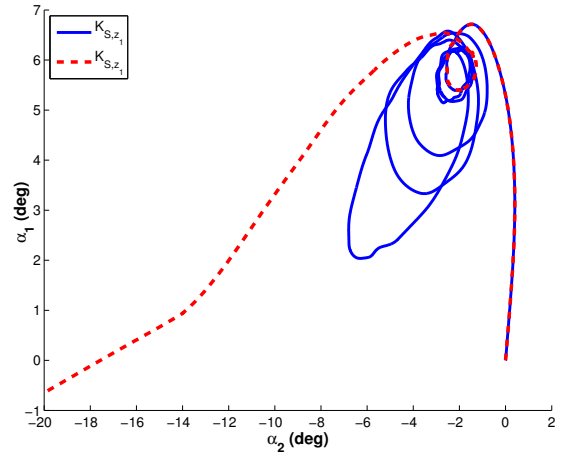

Fig. 8. Simulation results - Lateral side-slip angles

the stability of the system. However, the controller $K_{S, z_{2}}$ does not stabilize the system and its time responses leave the operation range.

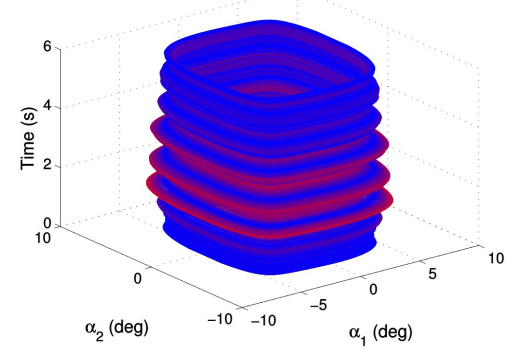

(a) Controller $K_{S, z_{1}}$

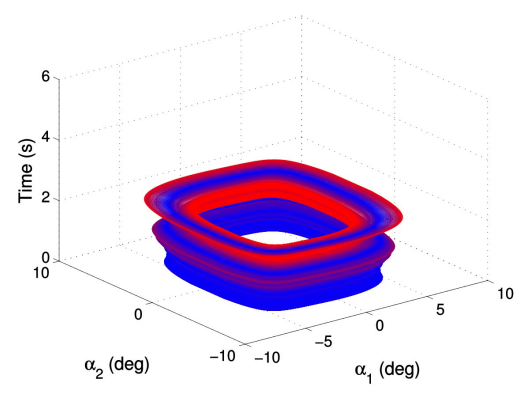

(b) Controller $K_{S, z_{2}}$

Fig. 9. Maximum Controlled Invariant Sets

Finally, the variation of the Maximum Controlled Invariant Set during the cruising of the vehicle is illustrated in Figure 9. In the figures the blue color represents the sets which are close to the nominal vertical force, while the red sets are related to the high variation. Comparing the sets it can be stated that the controller $K_{S, z_{1}}$ results in a smooth surface, while in the case of $K_{S, z_{2}}$ there are wide and narrow parts. The narrow parts are hazardous in the cruising, because the steering system has a low efficiency on the controllability of the vehicle under these circumstances, see e.g. the simulation time $t=1.5 \mathrm{~s}$.

\section{Conclusions}

The paper has analyzed the interaction between the vertical tire load and the lateral force. The nonlinear characteristics of the lateral tire force are approximated by the polynomial form. The Maximum Controlled Invariant Sets of the vehicle are calculated as functions of vertical tire loads. The size of the invariant sets decreases if the vertical tire force decreases. The invariant sets vary significantly if the vertical loads are different at the front and the rear. In the design of suspension control the vertical tire loads are in the focus. Weighting functions are defined by using the operation range of the controllers and the variation of the vertical tire loads. Thus, the vertical tire load is built into the suspension control. The design of the semi-active suspension control is based on the LPV method and the preview information.

\section{REFERENCES}

[1] T. Gillespie, Fundamentals of vehicle dynamics. Society of Automotive Engineers Inc, 1992.

[2] H. B. Pacejka, Tyre and vehicle dynamics. Oxford: Elsevier Butterworth-Heinemann, 2004.

[3] R. Rajamani, "Vehicle dynamics and control," Springer, 2005.

[4] P. Parrilo, "Semidefinite programming relaxations for semialgebraic problems," Mathematical Programming Ser. B, vol. 96, no. 2, pp. 293320, 2003.

[5] S. Prajna, A. Papachristodoulou, and F. Wu., "Nonlinear control synthesis by sum of squares optimization: A lyapunov-based approach," In Proceedings of the 5th IEEE Asian Control Conference, vol. 1, pp. 157-165, 2004.

[6] S. Fergani, O. Sename, and L. Dugard, "An $L P V / \mathcal{H}_{\infty}$ integrated vehicle dynamic controller," IEEE Transactions on Vehicular Technology, no. 4, pp. 1880-1889, 2016.

[7] B. Németh, P. Gáspár, and T. Péni, "Nonlinear analysis of vehicle control actuations based on controlled invariant sets," Int. Journal of Applied Mathematics and Computer Science.

[8] C. C. de Wit, H. Olsson, K. J. Astrom, and P. Lischinsky, "A new model for control of systems with friction," IEEE Transactions on Automatic Control, vol. 40, no. 3, pp. 419-425, 1995.

[9] U. Topcu, A. Packard, and P. Seiler, "Local stability analysis using simulations and sum-of-squares programming," Automatica, vol. 44, pp. 2669-2675, 2008.

[10] Z. Jarvis-Wloszek, R. Feeley, W. Tan, K. Sun, and A. Packard, "Some controls applications of sum of squares programming," Proceedings of 42nd IEEE Conference on Decision and Control, Maui, USA, vol. 5, pp. 4676-4681, 2003.

[11] E. D. Sontag, "A "universal" construction of Artstein's theorem on nonlinear stabilization," Systems \& Control Letters, vol. 13, pp. 117123, 1989.

[12] P. Gáspár and B. Németh, "Nonlinear analysis of actuator interventions using robust controlled invariant sets," 24th International Symposium on Dynamics of Vehicles on Road and Tracks. Graz, Austria, 2015.

[13] S. Savaresi, C. Poussot-Vassal, C. Spelta, O. Sename, and L. Dugard, Semi-Active Suspension Control for Vehicles. Elsevier - Butterworth Heinemann, 2010.

[14] E. Guglielmino, T. Sireteanu, C. W. Stammers., G. Ghita, and M.Giuclea, Semi-active suspension control. Springer, 2008.

[15] M. Canale, M. Milanese, C. Novara, and Z. Ahmad, "Semi-active suspension control using fast model predictive techniques," IEEE, Control System Technology, 2006.

[16] A. Do, C. Poussot-Vassal, O. Sename, and L. Dugard, "LPV control approaches in view of comfort improvement of automotive suspensions."

[17] J. Bokor and G. Balas, "Linear parameter varying systems: A geometric theory and applications," 16th IFAC World Congress, Prague, 2005.

[18] A. Packard and G. Balas, "Theory and application of linear parameter varying control techniques," American Control Conference, Workshop I, Albuquerque, New Mexico, 1997. 